\title{
6th Krefeld Skin Protection Meeting
}

Zum 6. Male trafen sich am 18. und 19. Juni 2002 in Krefeld rund 650 technische Aufsichtsbeamte, Sicherheitsfachkräfte, Vertreter der Berufsgenossenschaften, Arbeitsmediziner, berufsdermatologisch interessierte Hautärzte sowie betriebswirtschaftlich geprägte Fachleute, um über den Hautschutz in der täglichen Arbeitspraxis zu diskutieren. Die Veranstaltung wurde wieder gemeinsam von der Dermatologischen Klinik des Klinikums Krefeld und dem Geschäftsgebiet Hautschutz der Krefelder Firma Stockhausen GmbH \& Co. KG ausgerichtet.

Bereits zum 2. Male ging der Veranstaltung ein berufsgenossenschaftliches Forum voraus, das von Dr. Peter Kleesz, Berufsgenossenschaft Nahrungsmittel, Mannheim, Vorsitzender des Arbeitskreises Hautschutz, veranstaltet wurde.

Einer akademischen Tradition folgend wurde auf der Eröffnungsveranstaltung erneut der Krefelder Hautschutzpreis verliehen. Er ging diesmal an den Privatdozenten Dr. Swen-Malte John von der Universität Osnabrück, der für seine Habilitationsschrift „Klinische und experimentelle Untersuchungen zur Diagnostik in der Berufsdermatologie“ ausgezeichnet wurde.

Nach der Preisverleihung hielt Professor Dr. Reinhard Breit, München, einen Festvortrag über „Problem oder Propaganda: Lanolin- und Duftstoffallergie“.

Reinhard Breit deckte die Widersprüche der vorliegenden Studien zur Lanolinallergie akribisch auf und schloss sich uneingeschränkt der Aussage von Albert Montgomery Kligman an: Die Lanolinallergie ist ein Mythos, geschaffen hauptsächlich von überenthusiastischen professionellen Testern. Bezüglich der Duftstoffallergie, die insbesondere von skandinavischen Autoren als großes Gesundheitsproblem geschildert wird, erläuterte Reinhard Breit überzeugend seine gegenteilige Meinung.
Nach der Eröffnungsveranstaltung erfolgte eine Vortragsreihe zum Thema „Neues zum beruflichen Hautschutz“, die von Professor Dr. Thomas Diepgen, Ärztlicher Direktor am Institut für Arbeits- und Sozialmedizin, Universitäts-Klinikum Heidelberg, und Apotheker Dr. Joachim Kresken, Viersen, moderiert wurde. Dr. Karl-Christian Appl, Hautschutzzentrum Berlin, referierte zum Thema „Hauterkrankungen und deren Prävention im Tätigkeitsbereich Lebensmitteleinzelhandel“. Der Vortragende wies darauf hin, dass Handekzeme in der Branche Ernährung/Fleischer eine Erkrankungshäufigkeit von 2,9 Neuerkrankungen pro 1000 Beschäftigte und Jahr haben. Ganz besonders hautgefährdend ist die Tätigkeit im Frischebereich mit dem Verkauf von Fleisch-, Wurst-, Käse- und Fischwaren. Fast die Hälfte der BK5101-Verdachtsfälle resultiert aus dieser Tätigkeit. Das chronisch-irritative Handekzem und die beruflich verschlimmerte atopische Dermatitis gehören zu den häufigsten Erkrankungen. Die wichtigsten, potenziell schädigenden Faktoren sind ungeschützte Feuchtarbeit oder auch übertriebene Okklusion durch zu langes Tragen von Handschuhen.

Über neue Schutzprinzipien beim Hautschutz berichtete Andreas Klotz von der Firma Stockhausen. Er wies darauf hin, wie schwierig es ist, zwischen dem Hautschutz vor einer Schädigung zu unterscheiden und der Unterstützung der natürlichen Hautregeneration nach einer Schädigung, was eher einer Hautpflege entsprechen würde. Dabei berichtete er über erste Anwendungen einer kombinierten Testmethode. Seinen Ausführungen zufolge ist die Unterstützung der Hautregeneration bereits unter Arbeitsbelastung ein neuer Ansatz zur Gesunderhaltung arbeitender Haut und stellt keinen Widerspruch zum derzeitigen 3-Stufen-Modell des Hautschutzes dar.

Zum Schluss des wissenschaftlichen Programmes des 1 . Tages referierte Dr. Reinhold Rühl, Bau-BG Frankfurt, über Allergien 
durch beruflichen Hautschutz. Er wies insbesondere auf die Arbeitsgruppe „Schutzkleidung bei Umgang mit Chemikalien“ des Arbeitskreises „Dermale Exposition“ des UA IV des AGS hin. Diese hat inzwischen eine Liste der Allergene in Schutzhandschuhen, die auf dem deutschen Markt erhältlich sind, zusammengestellt. Sie ist im Internet unter www.gisbau.de in der Rubrik „Aktuelles" einsehbar.

Die 2. Vortragsserie wurde von Dr. Peter Kleesz und Professor Dr. Dr. Hans-Joachim Schwanitz, Universität Osnabrück, Arbeitsgruppe Gesundheitswissenschaften, moderiert. Zum Thema Hautschutz und Handschuhe referierte Dr. Annette zur Mühlen, Firma Stockhausen. Sie berichtete über Interaktionen von Hautschutzprodukten, die unter Schutzhandschuhen angewendet werden können. Diese möglichen Interaktionen werden durch Messung, z. B. der Dichtheit und Reißkraft, von medizinischen Einmalhandschuhen geprüft. Die Autorin berichtete über Prüfergebnisse mit einem Hautschutzgel und konnte nachweisen, dass durch dieses Gel nur eine sehr geringe Beeinflussung der beiden untersuchten Latexhandschuhtypen nachgewiesen wurde. Im Anschluss an diesen Vortrag referierte Dr. Martina Herrmann, Firma Stockhausen, zu Nutzung von 3-D-Hautmodellen zur Beurteilung der Wirksamkeit von Hautpflegeprodukten. Das von ihr dargestellte 3-D-Hautmodell wurde aus menschlichen Hautzellen gezüchtet und war mit menschlicher Haut in Ultrastruktur sowie in Lipid- und Enzymzusammensetzung vergleichbar.

Danach trug Professor Dr. Thomas L. Diepgen über Vorschriften zur Vorsorgeuntersuchung bei Hauterkrankungen vor. Er unterstrich die besondere Bedeutung der Prävention, da die Prognose einer bereits eingetretenen, berufsbedingten Hauterkrankung schlecht ist. Im Rahmen einer solchen Prävention stellen Vorsorgeuntersuchungen einen wichtigen Baustein dar. Leider wird bisher das Jugendarbeitschutzgesetz seiner präventivmedizinischen Rolle nicht gerecht und der für Berufe mit Hautbelastung vorgesehene berufsgenossenschaftliche Grundsatz G24 ist noch nicht rechtsverbindlich. Er berichtete des Weiteren über ein Konzept, welches vorsieht, bei der Novellierung der Gefahrstoffverordnung Voruntersuchungen für folgende Gefährdungen einzuführen: Arbeiten im feuchten Milieu, Exposition gegenüber Epoxidharzsystemen, Nickel oder seine Verbindungen sowie Chrom-6-Verbindungen. Entscheidend ist dabei, dass dem Arbeitsmediziner und Dermatologen Anhaltspunkte für Vorsorgeuntersuchungen von Versicherten, die beruflich einem erhöhten Hauterkrankungsrisiko ausgesetzt sind, gegeben werden, die den Gedanken der Prävention und nicht der Selektion in den Vordergrund stellen.

Die pädagogischen Aspekte des Hautschutzes trug Privatdozentin Dr. Britta Wulfhorst, Universität Osnabrück, Fachbereich Psychologie und Gesundheitswissenschaften, vor. Eine rein medizinisch-naturwissenschaftliche Sicht reicht ihrer Meinung nach nicht aus, um effektive Prävention zu betreiben, da das Gesamtspektrum von Bedingungen und Faktoren gesundheitsrelevanter Verhaltensweisen damit nicht erfasst werden kann. Die Effektivität und auch Effizienz von Präventionsprogrammen, die auf einer Integration dermatologischer und pädagogischer Interventionen basieren, ist in kontrollierten Studien mittlerweile eindeutig belegt worden. Voraussetzung für den Erfolg solcher Programme ist eine umfassende theoretische und praktische Schu- lung der Patienten, basierend auf grundlegenden pädagogisch didaktischen Prinzipien.

Über die geplante TRGS Dermale Exposition berichteten Dr. Reinhold Rühl, Bau-BG Frankfurt, und Wolfgang Stroh, Bau-BG Hannover. Zum Zeitpunkt des Hautschutztages gab es noch keinen diskussionsfähigen Entwurf der TRGS Dermale Exposition. Eine Rahmen-TRGS sollte aber in den Branchen tätigkeitsbezogene Umsetzungen ermöglichen. Zusätzlich sollen Gefährdungsbeurteilungen und Ersatzstoffsuche bei dermaler Exposition beschrieben werden. Empfehlungen zu Handschuh und Hautschutz werden eher allgemein ausfallen, da es den Branchen vorbehalten bleiben wird, die für sie jeweils sinnvollen Vorgehensweisen zu beschreiben. Angestrebt wird ein Schutzstufenkonzept.

Anmerkungen zu dieser geplanten TRGS Dermale Exposition machte im Anschluss daran Dr. Joachim Kresken, Viersen. Da der generelle Nutzen von Hautschutz unbestritten ist, sollte die TRGS seine Bedeutung als Arbeitsschutzmaßnahme bei dermaler Exposition gegenüber hautschädigenden Arbeitsstoffen betonen und grundsätzliche Anforderungen an die zum Einsatz kommenden Präparate definieren. Dazu gehört insbesondere die Notwendigkeit zur Durchführung von Wirksamkeitsnachweisen. Hierzu sollten Leitlinien erstellt werden und Zertifizierungsverfahren entwickelt werden. In der Diskussion war man sich darüber einig, dass die vorhandenen TRGS nicht ausreichend sind und deswegen eine TRGS Dermale Exposition anzustreben ist.

Zum Schluss des Hautschutztages fand wie immer eine Podiumsdiskussion unter der Moderation des Journalisten Kaspar Müller-Bringmann zur Thematik Akzeptanz von Hautschutz statt. Auf dem Podium saßen: Professor Dr. Thomas L. Diepgen, Dr. Ulrich Funke, Werksarzt Audi AG, Ingolstadt, Peter Kuhn, Sicherheitsbeauftragter der Aventis Pharma, Frank Küsters, Vertriebsleiter Inland Stockhausen GmbH \& Co. KG, und Privatdozentin Dr. Britta Wulfhorst. Die Experten und Diskussionsteilnehmer waren sich einig darin, dass der berufliche Hautschutz bei allen Fortschritten, die in den letzten Jahren erreicht wurden, noch sehr verbesserungsfähig ist. So haben besser strukturierte Vorsorgeuntersuchungen noch ein großes, ungenutztes Präventionspotenzial. Gleiches gilt für die noch zu formulierende neue TRGS Dermale Exposition. Auch bezüglich der Wirksamkeitsnachweise persönlicher Schutzmaßnahmen bleibt noch einiges zu tun. In der Diskussion wurde noch einmal ganz deutlich, dass beruflicher Hautschutz ohne angewandte Gesundheitspädagogik nicht funktionieren kann.

Die Kurzfassungen der Vorträge sind im Internet unter www.krefelder-hautschutztag.de/index.html einsehbar. 